\title{
RESEARCH
}

\section{Period functions associated to real-analytic modular forms}

\author{
Nikolaos Diamantis (1D and Joshua Drewitt
}

* Correspondence:

nikolaos.diamantis@nottingham.ac.uk University of Nottingham,

Nottingham, UK

\begin{abstract}
We define L-functions for the class of real-analytic modular forms recently introduced by F. Brown. We establish their main properties and construct the analogue of period polynomial in cases of special interest, including those of modular iterated integrals of length one.
\end{abstract}

\section{Introduction}

A well-known difference between the behaviour of holomorphic cusp forms and that of Maass cusp forms is that the former are "motivic" whereas the latter are, in general, not expected to be. This difference is fundamental for applications to arithmetic, and it is reflected in the possibility of proving results such as Manin's Periods Theorem. We state it here in a slightly weakened form to make the comparison with one of our theorems easier:

Proposition 1.1 [15] Letf be a cusp form of weight $k$ for $\Gamma:=S L_{2}(\mathbb{Z})$ which is a normalised eigenfunction of the Hecke operators. Let $L_{f}^{*}$ denote the "completed" $L$-function off. Then there are $\omega^{+}(f), \omega^{-}(f) \in \mathbb{C}$ such that

$$
\begin{aligned}
& L_{f}^{*}(j) \in \omega^{+}(f) K_{f}, \text { for odd } j \in\{2, \ldots k-2\} \text { and } L_{f}^{*}(j) \in \omega^{-}(f) K_{f}, \\
& \quad \text { for even } j \in\{2, \ldots k-2\} .
\end{aligned}
$$

where $K_{f}$ is the field generated by the Fourier coefficients off.

A key tool used to prove Proposition 1.1 was the period polynomial. This encodes the "critical values" of $L$-functions of cusp forms in an algebraically convenient way (Eichler cohomology). Period polynomials have been studied extensively, from various standpoints since at least the 70s and have been used to prove many important results besides Manin's Periods Theorem.

By contrast, the analogue of period polynomials for Maass cusp forms proved to be harder to construct. It was introduced and studied by Lewis and Zagier in the late 90s $[13,14]$. This period function found important uses in various contexts, though not in arithmetic applications.

(c) The Author(s) 2020. This article is licensed under a Creative Commons Attribution 4.0 International License, which permits use, sharing, adaptation, distribution and reproduction in any medium or format, as long as you give appropriate credit to the original author(s) and the source, provide a link to the Creative Commons licence, and indicate if changes were made. The images or other third party material in this article are included in the article's Creative Commons licence, unless indicated otherwise in a credit line to the material. If material is not included in the article's Creative Commons licence and your intended use is not permitted by statutory regulation or exceeds the permitted use, you will need to obtain permission directly from the copyright holder. To view a copy of this licence, visit http://creativecommons.org/licenses/by/4.0/. 
Against this contrasting picture, Brown [3-5] identified a "hybrid" space, which, though infinite dimensional and consisting of non-holomorphic functions, does appear to be "motivic". This is the space of modular iterated integrals, denoted by $\mathcal{M I}^{!}$. This, in turn, sits naturally in the broader class of real-analytic modular forms. The definitions are given in Sect. 2, but examples of modular iterated integrals include $\mathcal{E}_{2,0} \mathcal{E}_{0,2}+\frac{1}{2} \mathcal{E}_{1,1}^{2}$, where $\mathcal{E}_{r, s}(z)(r, s \in \mathbb{N})$ is the real-analytic Eisenstein series of Sect. 2.2, as well as other linear combinations of products of $\mathcal{E}_{r, s}$ and powers of $\operatorname{Im}(z)$. A genuinely new function in the space of modular iterated integrals is the function $F_{1,1}^{(1)}$ which satisfies

$$
(\Delta+2) F_{1,1}^{(1)}(z)=32 \pi^{3} \operatorname{Im}(z)^{3} \mathbb{G}_{4}(z) \overline{\mathbb{G}_{4}(z)}
$$

where $\Delta$ is the Laplacian (5) and $\mathbb{G}_{4}$ the holomorphic Eisenstein series with constant term $\frac{1}{240}$. Conjecturally, $F_{1,1}^{(1)}$ provides an explicit expression of an important modular graph function, whose theory was another motivation for defining real-analytic modular forms (see [3], \$9.3).

Brown provided evidence for the motivic nature of modular iterated integrals by proving interesting structural theorems and algebraicity and rationality results for Fourier coefficients of elements of $\mathcal{M} I^{!}$. In this paper, we give further credence to the motivic nature of the space of modular iterated integrals by defining $L$-functions in $\mathcal{M I}^{!}$(and, in fact, in the full space of real-analytic modular forms) and period functions.

The precise construction of $L$-functions in the space $\mathcal{M}_{r, s}^{!}$of real-analytic modular forms of weight $r, s$ is found in Sect. 3.1, but in the special case of "cuspidal" $f$, it is given by

$$
\begin{aligned}
L_{f}^{*}(w):= & \int_{1}^{\infty} \tilde{f}(i t) t^{w-1} \mathrm{~d} t+\int_{1}^{-\infty} \tilde{f}(i t) t^{w-1} \mathrm{~d} t \\
& +i^{r-s}\left(\int_{1}^{\infty} \tilde{f}(i t) t^{r+s-w-1} \mathrm{~d} t+\int_{1}^{-\infty} \stackrel{\circ}{ }(i t) t^{r+s-w-1} \mathrm{~d} t\right)
\end{aligned}
$$

where $\tilde{f}$ and $\stackrel{\circ}{f}$ stand for the "pieces" of $f$ that are, respectively, exponentially decreasing and exponentially increasing at infinity. The integral from 1 to $-\infty$ is explained in (14).

It is not obvious how to define period functions in $\mathcal{M}_{r, s}^{!}$appropriately. This is not surprising because period functions are normally supposed to reflect arithmeticity and the full space $\mathcal{M}_{r, s}^{!}$is too large to be of arithmetic nature in its entirety. However, here we show that, in the class $\mathcal{M I}_{1}^{!}$of modular iterated integrals of length 1 , it is possible to define period functions and, indeed, polynomials. The definition (Sect. 4) requires some preparation, so we will illustrate the construction here under a simplifying assumption that does not hold in general.

Fix $s, r \in \mathbb{N}$ of the same parity. For an integer $k \leq s, r$, a $\zeta \in \mathbb{C}$ and a smooth $g: \mathfrak{H} \cup \overline{\mathfrak{H}} \rightarrow$ $\mathbb{C}$, where $\mathfrak{H}$ (resp. $\overline{\mathfrak{H}}$ ) denote the upper (resp. lower) half-plane, we define the differential 1 -form

$$
\begin{aligned}
\omega_{k}(g ; \zeta)= & y^{k-1}\left(\frac{\partial g}{\partial z}+r g(z)\right)(\zeta-\bar{z})^{s-k}(\zeta-z)^{r-k} \mathrm{~d} z+(s-k) y^{k-1} \\
& \times g(z)(\zeta-\bar{z})^{s-k-1}(\zeta-z)^{r-k+1} \mathrm{~d} \bar{z}
\end{aligned}
$$

where $z=x+i y$.

Let now $F \in \mathcal{M}_{r, s}^{!}$which is a modular iterated integral (of length 1). As shown in [5], it can be uniquely decomposed as

$$
F=F_{0}+\cdots+F_{\min (r, s)}
$$


where $F_{k} \in \mathcal{M}_{r, s}^{!}$is an eigenfunction of the Laplacian with eigenvalue $(k-1)(r+s-k)$. Our simplifying assumption, for the purposes of the introduction, is that each $F_{k}$ has a vanishing "constant term" (see Sect. 2). We then set

$$
v_{k}(\zeta)=\int_{\zeta}^{i \infty} \omega_{k}\left(\tilde{F}_{k} ; \zeta\right)+\int_{\zeta}^{-i \infty} \omega_{k}\left(\stackrel{\circ}{F}_{k} ; \zeta\right)
$$

We can now state

Definition 1.2 Let $F \in \mathcal{M}_{r, s}^{!}$which is a modular integral. With the above notation, the period function $P(\zeta)$ of $F$ is given by

$$
P(\zeta)=\sum_{k=0}^{\min (r, s)}\left(v_{k}(-1 / \zeta) \zeta^{r+s-2 k}-v_{k}(\zeta)\right) .
$$

We will briefly explain why this period function induces a cocycle which is consistent with the Eichler cocycle of standard modular forms: For each fixed $k \in\{0, \ldots, \min (r, s)\}$, let $\Gamma:=\mathrm{SL}_{2}(\mathbb{Z})$ act on the space $P_{r+s-2 k}(\mathbb{C})$ of polynomials of degree $\leq r+s-2 k$, via:

$$
(P \underset{2 k-r-s, 0}{\|} \gamma)(\zeta):=P(\gamma \zeta)(c \zeta+d)^{r+s-2 k} \text { for all } P \in P_{r+s-2 k}(\mathbb{C}),\left(\begin{array}{l}
* * \\
c d
\end{array}\right) \in \mathrm{SL}_{2}(\mathbb{Z}) .
$$

(The reason for the unusual notation $\|$ will become clearer later). With this action, the Eichler cohomology group is the group $H^{1}\left(\mathrm{SL}_{2}(\mathbb{Z}), P_{r+s-2 k}(\mathbb{C})\right)$.

For each fixed $k \in\{0, \ldots, \min (r, s)\}$, let the map $\sigma_{k}: \mathrm{SL}_{2}(\mathbb{Z}) \rightarrow P_{r+s-2 k}(\mathbb{C})$ be given by

$$
\sigma_{k}(\gamma)=\left(v_{k} \|_{2 k-r-s} \gamma\right)-v_{k}
$$

We have $P(\zeta)=\sigma_{1}(S)(\zeta)+\cdots+\sigma_{\min (r, s)}(S)(\zeta)$. Further, define $\tilde{\sigma}_{k}: \mathrm{SL}_{2}(\mathbb{Z}) \rightarrow P_{r+s-2 k}(\mathbb{C})$ as

$$
\tilde{\sigma}_{k}(\gamma)=\int_{i}^{\gamma^{-1} i} \omega_{k}\left(F_{k} ; \zeta\right)
$$

By comparison, the Eichler cocycle of a weight $k$ cusp form $f$ for $\Gamma$ is $\sigma(\gamma)=\int_{i}^{\gamma^{-1} i} \omega(f ; \zeta)$, with $\omega(f ; \zeta)=f(z)(z-\zeta)^{k-2} \mathrm{~d} z$. Further, the cocycle $\sigma_{k}$ induced by our $P(\zeta)$ is also consistent with the Eichler cocycle $\tilde{\sigma}_{k}$ in the following sense:

Proposition 1.3 The maps $\sigma_{k}, \tilde{\sigma}_{k}$ define 1-cocycles in $P_{r+s-2 k}(\mathbb{C})$. Their classes in the Eichler cohomology $H^{1}\left(S L_{2}(\mathbb{Z}), P_{r+s-2 k}(\mathbb{C})\right)$ coincide.

The connection with our $L$-function is provided by the following theorem (see Theorem 5.3).

Theorem 1.4 Assume that $r \equiv s \bmod 4$. Let $P^{\prime}(\zeta)$ denote the polynomial obtained from $P(\zeta)$ upon removing its constant and leading terms. Then,

$$
P^{\prime}(\zeta)=\sum_{k=0}^{\min (r, s)} \sum_{l=1}^{r+s-2 k-1} a_{k, l} L_{F_{k}}^{*}(k+l) \zeta^{l}
$$

for some explicit $a_{k, l} \in \mathbb{Q}(i)$.

Finally, as an additional evidence that our definition of $L$-function is the "right" one, we prove the following:

Theorem 1.5 The analogue of Proposition 1.1 for $f$ weakly holomorphic holds. 
This differs from the full Manin's Periods Theorem in that it does not say anything about the values at 1 and $k-1$. As confirmed by numerical experiments, the lack of $K_{f}$ proportionality of $L_{f}^{*}(1)$ (and of $L_{f}^{*}(k-1)$ ) with the other odd "critical values" seems to be genuine and not just due to any incompleteness of our proof.

The precise statement of Theorem 1.5 is Theorem 6.3. To prove it, we use an analogous identity to Theorem 1.4 and the algebraic de Rham theory of weakly holomorphic modular forms [7]. K. Bringmann has shown us how we can use an identity of [2] to deduce a statement which, combined with Theorem 1.4, implies Theorem 1.5.

\section{Real-analytic modular forms}

We start by recalling the definition of real-analytic modular forms. Let $\Gamma=\mathrm{SL}_{2}(\mathbb{Z})$ and set $S=\left(\begin{array}{cc}0 & -1 \\ 1 & 0\end{array}\right), T=\left(\begin{array}{ll}1 & 1 \\ 0 & 1\end{array}\right)$ and $U=T S=\left(\begin{array}{cc}1 & -1 \\ 1 & 0\end{array}\right)$. Suppose that $r, s$ are positive integers of the same parity. For a smooth $g: \mathfrak{H} \rightarrow \mathbb{C}$ and $\gamma \in \Gamma$, we define a function $f \|_{r, s} \gamma$ given by

$$
f \|_{r, s} \gamma(z)=j(\gamma, z)^{-r} j(\gamma, \bar{z})^{-s} f(\gamma z) \quad \text { for all } z \in \mathfrak{H}
$$

where

$$
j\left(\left(\begin{array}{cc}
* & * \\
c & d
\end{array}\right), z\right):=c z+d .
$$

We extend the action to $\mathbb{C}[\Gamma]$ by linearity. With this notation, we can make the following definition.

Definition 2.1 A real-analytic function $f: \mathfrak{H} \rightarrow \mathbb{C}$ is called real-analytic modular form of weights $(r, s)$ for $\Gamma$ if

1. for all $\gamma \in \Gamma$ and $z \in \mathfrak{H}$, we have $f \|_{r, s} \gamma=f$, i.e.,

$$
f(\gamma z)=j(\gamma, z)^{r} j(\gamma, \bar{z})^{s} f(z) \quad \text { for all } z \in \mathfrak{H} .
$$

2. for some $C>0, f(z)=O\left(e^{C y}\right)$ as $y \rightarrow \infty$, uniformly in $x$ (where $z=x+i y$ ). Further,

$$
f(z)=\sum_{|j| \leq M} y^{j}\left(\sum_{m, n \geq-N} a_{m, n}^{(j)} q^{m} \bar{q}^{n}\right)
$$

for some $M, N \in \mathbb{N}, a_{m, n}^{(j)} \in \mathbb{C}$. Here, $q=\exp (2 \pi i z)$.

We denote the space of real analytic modular forms of weights $(r, s)$ for $\Gamma$ by $\mathcal{M}_{r, s}^{!}$. We set $\mathcal{M}^{!}=\oplus_{r, s} \mathcal{M}_{r, s}^{!}$. This class of functions was introduced by Brown [3-5] whose initial motivation was related, on the one hand, to some non-holomorphic modular forms originating from iterated extensions of pure motives and with coefficients that are periods. On the other hand, he was motivated by open questions about modular graph functions appearing in string perturbation theory.

The space $\mathcal{M}^{!}$contains or intersects various previously studied classes of important modular objects and the point of view we adopt here is to consider real-analytic forms as a unifying tool for those classes. For example, when $s=0$, an element $f$ of $\mathcal{M}_{r, 0}^{!}$which is holomorphic in $\mathfrak{H}$ is a standard weakly holomorphic modular form of weight $r$ for $\Gamma$. We denote their space by $M_{r}^{!}$and set $M^{!}=\oplus_{r} M_{r}^{!}$. The space $M_{r}^{!}$contains, of course, the space $M_{r}$ (resp. $S_{r}$ ) of classical modular (resp. cusp) forms of weight $r$. 
When $r=0$ we are similarly led to the space $\bar{M}_{r}$ of weakly anti-holomorphic modular forms. Another subspace is $\mathcal{M}_{r, s}$, which is obtained upon imposing the condition that $a_{m, n}^{(k)}$ should vanish when $m$ or $n$ are negative. It was defined and studied in [3]. Their direct sum over all $r, s$ is denoted by $\mathcal{M}$.

The relation with Maass forms is more complicated. On the one hand, the definition of $\mathcal{M}$ ! allows for forms which are not eigenfunctions of the Laplacian, but, on the other, it requires a more restrictive form of a Fourier expansion than that of Maass forms. We will exploit this relation in the sequel in order to define some of our main objects, and, in particular, we will see that constructions from the theory of Maass cusp forms will be the basis for period functions of certain elements of $\mathcal{M}$ !

The Fourier expansion (1) can be uniquely decomposed into a sum of an "principal part" $\stackrel{\circ}{f}$, an exponentially decaying part $\tilde{f}$ and the "constant term" $f^{0}$ defined as follows:

$$
\begin{aligned}
\tilde{f}(z) & :=\sum_{|j| \leq M} y^{j}\left(\sum_{\substack{m, n \geq-N \\
m+n>0}} a_{m, n}^{(j)} q^{m} \bar{q}^{n}\right) \\
f^{0}(z) & :=\sum_{|j| \leq M} y^{j}\left(\sum_{\substack{m, n \geq-N \\
m+n=0}} a_{m, n}^{(j)} q^{m} \bar{q}^{n}\right) \quad \text { and } \\
\stackrel{f}{f}(z) & :=f(z)-\tilde{f}(z)-f^{0}(z)
\end{aligned}
$$

\subsection{Eigenforms for the Laplacian}

The Lie algebra $\mathfrak{s l}_{2}$ acts on $\mathcal{M}^{!}$via the Maass operators $\partial_{r}: \mathcal{M}_{r, s}^{!} \rightarrow \mathcal{M}_{r+1, s-1}^{!}$and $\bar{\partial}_{s}: \mathcal{M}_{r, s}^{!} \rightarrow \mathcal{M}_{r-1, s+1}^{!}$given by

$$
\partial_{r}=2 i y \frac{\partial}{\partial z}+r \quad \text { and } \quad \bar{\partial}_{s}=-2 i y \frac{\partial}{\partial \bar{z}}+s .
$$

They induce bigraded derivations on $\mathcal{M}^{!}$denoted by $\partial$ and $\bar{\partial}$, respectively.

The Laplacian $\Delta_{r, s}: \mathcal{M}_{r, s}^{!} \rightarrow \mathcal{M}_{r, s}^{!}$is defined by

$$
\Delta_{r, s}=-\bar{\partial}_{s-1} \partial_{r}+r(s-1)=-\partial_{r-1} \bar{\partial}_{s}+s(r-1) .
$$

It induces a bigraded operator $\Delta$ of bidegree $(0,0)$ on $\mathcal{M}$ !

An operator which is essentially equivalent to $\Delta_{r, s}$ but which is more convenient for some computations in the sequel is

$$
\Omega_{k}=-y^{2}\left(\frac{\partial^{2}}{\partial x^{2}}+\frac{\partial^{2}}{\partial y^{2}}\right)+i k y \frac{\partial}{\partial x} .
$$

When working with $\Omega_{k}$, the following version of the "stroke" operator will be more appropriate to work with than $\|$. Specifically, for $\gamma \in \Gamma$ and $f: \mathfrak{H} \rightarrow \mathbb{C}$, the function $\left.f\right|_{k} \gamma$ is defined by

$$
\left(\left.f\right|_{k} \gamma\right)(z)=\left(\frac{j(\gamma, z)}{|j(\gamma, z)|}\right)^{-k} f(\gamma z) \text { for all } z \in \mathfrak{H} .
$$

We extend the action to $\mathbb{C}[\Gamma]$ by linearity. To move between the $\Delta$ to the $\Omega$ formalism, the following lemma will be useful: 
Lemma 2.2 If, for some $r, s \in \mathbb{Z}$ of the same parity, $F$ is an element of $\mathcal{M}_{r, s}^{!}$such that $\Delta_{r, s} F=\lambda F$ for some $\lambda \in \mathbb{R}$, then $F_{1}:=y^{\frac{r+s}{2}} F$ satisfies

$$
\left.F_{1}\right|_{r-s} \gamma=F_{1} \quad \text { for all } \gamma \in \Gamma \text { and } \quad \Omega_{r-s} F_{1}=\left(\lambda+\left(\frac{r+s}{2}\right)\left(1-\frac{r+s}{2}\right)\right) F_{1} .
$$

Proof We observe that

$$
\Delta_{r, s}=\Omega_{r-s}-(r+s) y \frac{\partial}{\partial y}
$$

and that, for each smooth $f: \mathfrak{H} \rightarrow \mathbb{C}$,

$$
\left.f\right|_{k} \gamma=f \underset{\frac{k}{2},-\frac{k}{2}}{\|} \gamma \text { and }\left.\left(y^{\frac{r+s}{2}} f\right)\right|_{r-s} \gamma=y^{\frac{r+s}{2}}\left(f \|_{r, s} \gamma\right) \text { for all } \gamma \in \Gamma .
$$

An easy computation then implies the lemma.

For $\lambda \in \mathbb{R}$, set

$$
\mathcal{H} \mathcal{M}^{!}(\lambda):=\operatorname{Ker}\left(\Delta-\lambda: \mathcal{M}^{!} \rightarrow \mathcal{M}^{!}\right) .
$$

Also set $\mathcal{H} \mathcal{M}^{!}:=\oplus_{\lambda} \mathcal{H} \mathcal{M}^{!}(\lambda)$. The following lemma summarises some of the special features of the Fourier expansions of $f \in \mathcal{H} \mathcal{M}^{!}(\lambda)$.

Lemma 2.3 [5] Let $f \in \mathcal{H M}^{!}(\lambda) \cap \mathcal{M}_{r, s^{*}}^{!}$Then, $\lambda \in \mathbb{Z}$ and there is a $k_{0} \in \mathbb{Z}$ such that $k_{0}<1-r-s-k_{0}$ and $\lambda=k_{0}\left(1-r-s-k_{0}\right)$. There are unique $f^{h}, f^{a}$ of the form

$$
\begin{aligned}
f^{h}(z) & =\sum_{j=k_{0}}^{-s} y^{j} \sum_{\substack{m \geq-N \\
m \neq 0}} a_{m}^{(j)} q^{m} \\
f^{a}(z) & =\sum_{j=k_{0}}^{-r} y^{j} \sum_{\substack{m \geq-N^{\prime} \\
m \neq 0}} b_{m}^{(j)} \bar{q}^{m}
\end{aligned}
$$

$\left(a_{m}^{(j)}, b_{m}^{(j)} \in \mathbb{C}\right.$ and $\left.N, N^{\prime} \in \mathbb{N}\right)$ such that

$$
f=f^{0}+f^{h}+f^{a} .
$$

Furthermore, the constant term has the form $f^{0}(z)=a y^{k_{0}}+b y^{1-r-s-k_{0}}$ for some $a, b \in \mathbb{C}$. Finally, $f^{h}, f^{a}, y^{k_{0}}$ and $y^{1-r-s-k_{0}}$ are eigenfunctions of $\Delta_{r, s}$ with eigenvalue $\lambda$.

Proof Lemma 4.3 of [5] together with the remarks following it.

\subsection{Real analytic Eisenstein series}

An example of an element of $\mathcal{M}_{r, s}^{!}$, and, indeed, of $\mathcal{M}_{r, s}$, which is, in addition, an eigenform for the Laplacian is the non-holomorphic Eisenstein series. Specifically, in a normalisation and notation which is more appropriate for the theory of real-analytic forms, a real analytic Eisenstein series $\mathcal{E}_{r, s}$ is given for $r, s \in \mathbb{N}$ and $z \in \mathfrak{H}$ by

$$
\mathcal{E}_{r, s}(z)=\frac{1}{2} \sum_{\gamma \in B \backslash \Gamma} \frac{\operatorname{Im}(z)}{j(\gamma, z)^{r+1} j(\gamma, \bar{z})^{s+1}}
$$

where $B$ is the subgroup of translations. This series converges absolutely and belongs to $\mathcal{M}_{r, s}$ It further has a meromorphic continuation to the entire complex plane and is an eigenfunction of $\Delta$ with eigenvalue $-r-s$. 
Its Fourier expansion has been computed explicitly in Th. 3.1. of [11] and in Prop. 11.2.16 of [9]. We summarise it here and will see how it fits with Lemma 2.3. With the notation of that lemma,

$$
\begin{aligned}
\mathcal{E}_{r, s}(z)=\mathcal{E}_{r, s}^{0}(z)+\frac{\pi^{\frac{r+s+2}{2}}}{\Gamma(1+r) \zeta(r+s+1)}\left\{\sum_{j=-r-s}^{-s} y^{j} \sum_{m \geq 1} \frac{\sigma_{r+s+1}(m) \alpha_{-j-\frac{r+s}{2}}^{+}(4 \pi)^{j+\frac{r+s}{2}}}{m^{1-j}} q^{m}\right. \\
\left.+\sum_{j=-r-s}^{-r} y^{j} \sum_{m \geq 1} \frac{\sigma_{r+s+1}(m) \alpha_{-j-\frac{r+s}{2}}^{-}(4 \pi)^{j+\frac{r+s}{2}}}{m^{1-j}} \bar{q}^{m}\right\}
\end{aligned}
$$

where

$$
\alpha_{j}^{ \pm}:=(-1)^{j}\left(j+\frac{|r-s|}{2}\right) !\left(\begin{array}{c}
s+\frac{r-s}{2}+\frac{|r-s|}{2} \\
j+\frac{|r-s|}{2}
\end{array}\right)\left(\begin{array}{c}
( \pm 1-1) \frac{r-s}{2}-1-s \\
j \pm \frac{r-s}{2}
\end{array}\right) .
$$

Here $\left(\begin{array}{l}a \\ b\end{array}\right)$ with $a<0$ are defined in accordance with the convention that, if $a<0$ and $j \geq 0$, then $\left(\begin{array}{c}a+j \\ j\end{array}\right)=(a+j)(a+j-1) \ldots(a+1) / j$ !. Thus, $k_{0}=-r-s$ and

$$
\begin{aligned}
& a_{m}^{(j)}=\frac{(2 \pi)^{r+s+j} 2^{j} \pi \alpha_{-j-\frac{r+s}{2}}^{+}}{\Gamma(1+r) \zeta(r+s+1)} \sigma_{r+s+1}(m) m^{j-1} \text { and } \\
& b_{m}^{(j)}=\frac{(2 \pi)^{r+s+j} 2^{j} \pi \alpha_{-j-\frac{r+s}{2}}^{-}}{\Gamma(1+r) \zeta(r+s+1)} \sigma_{r+s+1}(m) m^{j-1}
\end{aligned}
$$

which is consistent with Lemma 2.3 .

\subsection{Modular iterated integrals of length one}

In [5], the space of modular iterated integrals is defined. We will consider only the special case of length one: The space $\mathcal{M} \mathcal{I}_{1}^{!}$of modular iterated integrals of length one is defined to be the largest subspace of $\mathcal{M}^{!}$which satisfies

$$
\begin{aligned}
& \partial \mathcal{M} \mathcal{I}_{1}^{!} \subset \mathcal{M} \mathcal{I}_{1}^{!}+M^{!}\left[y^{ \pm}\right] \\
& \bar{\partial} \mathcal{M} \mathcal{I}_{1}^{!} \subset \mathcal{M} \mathcal{I}_{1}^{!}+\overline{M^{!}}\left[y^{ \pm}\right]
\end{aligned}
$$

A characterisation of this space is provided in [5]:

Proposition 2.4 (Prop. 5.8 of [5]) Any element $F$ of $\mathcal{M I}_{1}^{!}$of weights $r$, s can be written uniquely as

$$
F=\sum_{k=0}^{\min (r, s)} F_{k}
$$

for some elements of $\mathcal{M} \mathcal{I}_{1}^{!}$of weights $r$, s such that $\Delta_{r, s} F_{k}=\lambda_{k} F_{k}$ where

$$
\lambda_{k}=(k-1)(r+s-k) .
$$

This, in particular, implies that the value of the invariant $k_{0}$ (see Lemma 2.3) for $F_{k}$ is

$$
k_{0}=k-r-s .
$$

We will interpret the functions $F_{k}$ of the last proposition in the setting of the last section. 
Proposition 2.5 Let $F$ be an element of $\mathcal{M} \mathcal{I}_{1}^{!}$with weights $r, s$ and let $F_{k}$ ( $k \in$ $\{0, \ldots, \min (r, s)\})$ be as in Proposition 2.4. Then $y^{\frac{r+s}{2}} F_{k}(z)$ is $\Gamma$-invariant under the action of $\left.\right|_{r-s}$ and is an eigenfunction of $\Omega_{r-s}$ with eigenvalue

$$
\frac{1}{4}-\mu_{k}^{2} \quad \text { where } \mu_{k}=-k+\frac{r+s+1}{2} .
$$

For $g=\tilde{F}_{k}, \stackrel{\circ}{F}_{k}, y^{k-r-s}, y^{1-k}$ [in the notation (2), (4)], we have

$$
\Omega_{r-s}\left(y^{\frac{r+s}{2}} g\right)=\left(\frac{1}{4}-\mu_{k}^{2}\right) y^{\frac{r+s}{2}} g
$$

Proof By Lemma 2.2 and Proposition 2.4, $y^{\frac{r+s}{2}} F_{k}(z)$ is $\Gamma$-invariant under the action of $\left.\right|_{r-s}$ and eigenfunction of $\Omega_{r-s}$ with eigenvalue

$$
\begin{aligned}
\lambda_{k}+\left(\frac{r+s}{2}\right)\left(1-\frac{r+s}{2}\right) & =(k-1)(r+s-k)+\left(\frac{r+s}{2}\right)\left(1-\frac{r+s}{2}\right) \\
& =\frac{1}{4}-\left(k-\frac{r+s+1}{2}\right)^{2} .
\end{aligned}
$$

To establish the eigen-properties (10), we apply the last statement of Lemma 2.3 to $F_{k} \in \mathcal{H} \mathcal{M}^{!}\left(\lambda_{k}\right)$. Then since $\Delta_{r, s} F_{k}^{h}=\lambda_{k} F_{k}^{h}$ and $\Delta_{r, s} F_{k}^{0}=\lambda_{k} F_{k}^{0}$, we have

$$
\left(\Delta_{r, s}-\lambda_{k}\right)\left(\sum_{j=k_{0}}^{-s} y^{j} \sum_{-N \leq m<0} a_{m}^{(j)} q^{m}\right)=-\left(\Delta_{r, s}-\lambda_{k}\right)\left(\sum_{j=k_{0}}^{-s} y^{j} \sum_{m>0} a_{m}^{(j)} q^{m}\right)
$$

Let $\mathcal{P}$ be the space of polynomials of $y$ over $\mathbb{C}$. By (2.22) of [3], we have

$$
\Delta_{r, s}\left(\mathcal{P} \cdot q^{m} \bar{q}^{n}\right) \subset \mathcal{P} \cdot q^{m} \bar{q}^{n}
$$

for each $m, n \in \mathbb{Z}$. Therefore, the LHS of (11) will be a polynomial in $q^{-1}$ with coefficients in $\mathcal{P}$, and thus, if not identically 0 , it will have exponential growth as $y \rightarrow \infty$. This is impossible because, by (12), the RHS of (11) decays exponentially as $y \rightarrow \infty$. Therefore, the LHS vanishes and

$$
\Delta_{r, s}\left(\sum_{j=k_{0}}^{-s} y^{j} \sum_{-N \leq m<0} a_{m}^{(j)} q^{m}\right)=\lambda_{k}\left(\sum_{j=k_{0}}^{-s} y^{j} \sum_{-N \leq m<0} a_{m}^{(j)} q^{m}\right) .
$$

We similarly see that $\sum_{j=k_{0}}^{-r} y^{j} \sum_{-N^{\prime} \leq m<0} b_{m}^{(j)} \bar{q}^{m}$ is an eigenfunction of $\Delta_{r, s}$ with eigenvalue $\lambda_{k}$. Thus, $\stackrel{\circ}{F}_{k}$ is an eigenfunction of $\Delta_{r, s}$ with eigenvalue $\lambda_{k}$.

Since $y^{k-r-s}, y^{1-k}$ are also eigenfunctions of $\Delta_{r, s}$ with eigenvalue $\lambda_{k}$, we deduce (with Lemma 2.2) the desired eigen-properties of $y^{(s+r) / 2} y^{k-r-s}$ and $y^{(s+r) / 2} y^{1-k}$. The eigenproperties of $y^{(s+r) / 2} \stackrel{\circ}{F}_{k}, y^{(s+r) / 2} y^{k-r-s}, y^{(s+r) / 2} y^{1-k}$, just proved, together with the eigenproperty of $y^{(s+r) / 2} F_{k}$ then imply the eigen-property of $y^{(s+r) / 2} \tilde{F}_{k}$.

\section{L-functions}

The obstacles to extending the definition of $L$-functions of standard modular forms to $\mathcal{H} \mathcal{M}^{!}$are due to the potentially exponential growth of functions in $\mathcal{M}^{!}$combined with the lack of holomorphicity. To tackle the former, we can give a definition that is based on the expression of standard $L$-functions through Mellin transforms. This will, in fact, allow us to define $L$-functions on the entire $\mathcal{M}$ ! 


\subsection{L-functions in $\mathcal{M}^{!}$}

Let $f \in \mathcal{M}_{r, s}^{!}$with an expansion (1). We let the implied logarithm take the principal branch of the logarithm and we set, for $w \neq-j, r+s+j(|j| \leq M)$,

$$
\begin{aligned}
L_{f}^{*}(w) & :=\left(\int_{1}^{\infty} \tilde{f}(i t) t^{w-1} \mathrm{~d} t+\int_{1}^{-\infty} f(i t) t^{w-1} \mathrm{~d} t-\sum_{|j| \leq M} \sum_{\substack{m, n \geq-N \\
m+n=0}} \frac{a_{m, n}^{(j)}}{w+j}\right) \\
& +i^{r-s}\left(\int_{1}^{\infty} \tilde{f}(i t) t^{r+s-w-1} \mathrm{~d} t+\int_{1}^{-\infty} f(i t) t^{r+s-w-1} \mathrm{~d} t-\sum_{\substack {|j| \leq M \\
\begin{subarray}{c}{m, n \geq-N \\
m+n=0{ | j | \leq M \\
\begin{subarray} { c } { m , n \geq - N \\
m + n = 0 } }\end{subarray}} \frac{a_{m, n}^{(j)}}{r+s-w+j}\right) .
\end{aligned}
$$

The rigorous meaning of the first integral from 1 to $-\infty$ is

$$
\sum_{|j| \leq M} \sum_{\substack{m, n \geq-N \\ m+n<0}} \frac{a_{m, n}^{(j)}}{(2 \pi(m+n))^{j+w}} \Gamma(j+w, 2 \pi(m+n))
$$

where $\Gamma(r, z)$ denotes the incomplete Gamma function

$$
\Gamma(r, z):=\int_{z}^{\infty} e^{-t} t^{r} \frac{\mathrm{d} t}{t} .
$$

For $z \neq 0$, this has an analytic continuation to the entire $r$-plane, and therefore, (14) is welldefined for all values of $w$ by the analytic continuation of incomplete Gamma function. By contrast, the real integral as written in (13) is not convergent at 0 unless $\Re(w)>1+M$. We interpret likewise the second integral from 1 to $-\infty$ in (13). The reason we preferred to write formally those terms as integrals was to stress the symmetry with the other terms and hint at the origin of the definition in a "regularisation" introduced in [6].

Since, in addition, $\tilde{f}$ decays exponentially at infinity, all integrals in (13) are well-defined. As mentioned above, the above construction was inspired by "regularisation" introduced in [6], Sect. 4. (See [10], for another application of this idea.)

The definition immediately implies the following:

Proposition 3.1 Let $f \in \mathcal{M}_{r, s}^{!}$(with $r \equiv s \bmod$ ). The L-function off is meromorphic with finitely many poles and satisfies

$$
L_{f}^{*}(w)=i^{r-s} L_{f}^{*}(r+s-w)
$$

for all w away from the poles.

In such generality, the definition is somewhat formal and would be unlikely to lead to arithmetic insight for all $f \in \mathcal{M}_{r, s}^{!}$. To obtain more refined information, we restrict to subspaces of $\mathcal{M}_{r, s}^{!}$.

We first note that, in the subspace $\mathcal{M}_{r, s}$ of $f \in \mathcal{M}_{r, s}^{!}$with moderate growth at infinity, our definition coincides with that of Sect. 9.4 of [3]. Specifically, in that case, $\stackrel{\circ}{f}=0$ and the Fourier coefficients of $f$ have polynomial growth. $\operatorname{For} \operatorname{Re}(w) \gg 0$, the change of variable $t \rightarrow 1 / t$ in the third integral of (13) together with the transformation law of $f$ implies that $L_{f}^{*}(w)$ coincides with the function $\Lambda(f, w)$ of Sect. 9.4. of [3]. See also, Section 9.4 of [4] where this construction is applied to the important subclass of $\mathcal{M}_{r, s}$ consisting of modular analogues of the single-valued polylogarithms. 


\subsection{L-functions in $\mathcal{H} \mathcal{M}^{!}(\lambda)$ and in $\mathcal{M} \mathcal{I}_{1}^{!}$}

Let $f \in \mathcal{H M}^{!}(\lambda) \cap \mathcal{M}_{r, s}^{!}$. Using the Fourier expansion of $f$ provided by Lemma 2.3, the general definition of $L_{f}^{*}(w)$ we gave above leads to an expression as a series. This is more natural because it is reminiscent of the original definition of $L$-series of standard modular forms and because, in the case of weakly holomorphic modular forms, it coincides with the $L$-functions already associated with such forms ([1] and references therein).

To ensure that the series we will eventually obtain converges absolutely, we need an analogue of the "trivial bound" about the Fourier coefficients. As in the case of weakly holomorphic forms ([8], Lemma 3.2), the growth is, in general, exponential. Although the proof parallels that of [8], there are some complications because of the presence of two weights and of the powers of $y$, so we present a full proof.

Proposition 3.2 Let $f \in \mathcal{H M}^{!}(\lambda)$. With the notation of Lemma 2.3, for each $j \in$ $\left\{k_{0}, \ldots,-s\right\}$ (resp. $\left.j \in\left\{k_{0}, \ldots,-r\right\}\right)$, there is a $C>0$ such that,

$$
a_{n}^{(j)} \ll e^{C \sqrt{n}}\left(\text { resp. } b_{n}^{(j)} \ll e^{C \sqrt{n}}\right) \quad \text { as } n \rightarrow \infty
$$

Proof Set $N_{0}=\max \left(N, N^{\prime}\right)$ and let $n>N_{0}$. Then we have

$$
\begin{aligned}
\int_{0}^{1} f(z) e^{-2 \pi i n z} \mathrm{~d} x= & \int_{0}^{1} f^{0}(z) e^{-2 \pi i n z} \mathrm{~d} x \\
& +\sum_{j=k_{0}}^{-s} y^{j} \sum_{\substack{m \geq-N \\
m \neq 0}} a_{m}^{(j)} \int_{0}^{1} e^{2 \pi i(m-n) z} \mathrm{~d} x \\
& +\sum_{j=k_{0}}^{-r} y^{j} \sum_{\substack{m \geq-N^{\prime} \\
m \neq 0}} a_{m}^{(j)} e^{2 \pi(n-m) y} \int_{0}^{1} e^{-2 \pi i(m+n) x} \mathrm{~d} x \\
& =\sum_{j=k_{0}}^{-s} y^{j} a_{n}^{(j)}
\end{aligned}
$$

since, for $n>N_{0}$ and $m \geq-N^{\prime}, m+n>0$. Likewise,

$$
\int_{0}^{1} f(z) e^{2 \pi i n z} \mathrm{~d} x=\sum_{j=k_{0}}^{-r} y^{j} b_{n}^{(j)} e^{-4 \pi n y} .
$$

Suppose that $-s>k_{0}$. Then (15) implies

$$
e^{2 \pi n y} y^{-k_{0}} \int_{0}^{1} f(z) e^{-2 \pi i n x} \mathrm{~d} x=\sum_{j=0}^{-s-k_{0}} y^{j} a_{n}^{\left(j+k_{0}\right)}
$$

and thus

$$
\left(-s-k_{0}\right) ! a_{n}^{(-s)}=\frac{\partial^{-s-k_{0}}}{\partial y^{-s-k_{0}}}\left(e^{2 \pi n y} y^{-k_{0}} \int_{0}^{1} f(z) e^{-2 \pi i n x} \mathrm{~d} x\right) .
$$

The RHS will be a sum of products of $e^{2 \pi n y}$, polynomials in $y$ and $n$ and

$$
\int_{0}^{1} \frac{\partial^{j} f(z)}{\partial y^{j}} e^{-2 \pi i n x} \mathrm{~d} x
$$

for $j \in\left\{0, \ldots,-s-k_{0}\right\}$. Now, we note that, for all $k, l$,

$$
\frac{\partial}{\partial y}=\frac{1}{2 y}\left(\partial_{k}+\bar{\partial}_{l}-k-l\right)
$$


and, since $y^{-1} \in \mathcal{M}_{1,1}^{!}$, we have $\frac{\partial}{\partial y}\left(\mathcal{M}^{!}\right) \subset \mathcal{M}^{!}$. Thus,

$$
\frac{\partial^{j}}{\partial y^{j}}\left(\mathcal{M}^{!}\right) \subset \mathcal{M}^{!}
$$

We will use this to bound (18) with the help of this lemma:

Lemma 3.3 For each $f \in \mathcal{M}_{r, s}^{!}$, there is a $C>0$ such that $f(z)=O\left(e^{C / y} y^{-(r+s) / 2}\right)$ as $y \rightarrow 0$, uniformly in $x$.

Proof The standard $j$-function $j(z)$ does not vanish in the interior of the standard fundamental domain $\mathcal{F}$ of $\Gamma$. Therefore, if $c>0$ is such that $f(z)=O\left(e^{c y}\right)$ as $y \rightarrow \infty$ (uniformly in $x)$, then $F(z)=\left|f(z) y^{\frac{r+s}{2}} j(z)^{-c-\epsilon}\right|$ is bounded in the standard fundamental domain $\mathcal{F}$ of $\Gamma$. On the other hand, $\left|f(z) y^{\frac{r+s}{2}}\right|$ is invariant under any $\gamma \in \Gamma$ and thus under $S$. This implies that there is a $C>0$, such that, as $y \rightarrow 0$,

$$
\left|f(z) y^{\frac{r+s}{2}}\right|=\left|f(-1 / z) \operatorname{Im}(-1 / z)^{\frac{r+s}{2}}\right|=O\left(e^{C \operatorname{Im}(-1 / z)}\right)=O\left(e^{C / y}\right)
$$

uniformly in $x$, which gives the result.

With this lemma and (20), we see that, for $y \rightarrow 0$,

$$
\frac{\partial^{j} f}{\partial y^{j}}=O\left(e^{C / y} y^{M}\right)
$$

for some constant depending on $r$, s. From (17), we then conclude $a_{n}^{(-s)} \ll e^{2 \pi n y} e^{C / y} y^{M_{1}} N^{M_{2}}$, where $C, M_{1}, M_{2}$ and the implied constant depend only on $r, s$ and $k_{0}$. For $y=1 / \sqrt{n}$, this implies the bound of the proposition in the case $j=-s$.

Next, we differentiate in $y$ both sides of

$$
\sum_{j=0}^{-s-k_{0}-1} y^{j} a_{n}^{\left(j+k_{0}\right)}=-y^{-s-k_{0}} a_{n}^{(-s)}+e^{2 \pi n y} y^{-k_{0}} \int_{0}^{1} f(z) e^{-2 \pi i n x} \mathrm{~d} x
$$

to get

$$
\begin{aligned}
\left(-s-k_{0}-1\right) ! a_{n}^{(-s-1)}= & -\left(-s-k_{0}\right) ! y a_{n}^{(-s)} \\
& +\frac{\partial^{-s-k_{0}-1}}{\partial y^{-s-k_{0}-1}}\left(e^{2 \pi n y} y^{-k_{0}} \int_{0}^{1} f(z) e^{-2 \pi i n x} \mathrm{~d} x\right) .
\end{aligned}
$$

Arguing as above for the second term, and using the bound for $a_{n}^{(-s)}$ we proved above, we deduce the bound for $j=-s-1$. Continuing in this way, we deduce the result for all $j$.

It is clear from the argument (essentially by interchanging the roles of $s$ and $k_{0}$ ), that it remains valid when $-s \leq k_{0}$.

To prove the bound for $b_{n}^{(j)}$, we work in the same way but based on (16), instead of (15).

We are now ready to use the Fourier expansion given in Lemma 2.3 to express the $L$-function of an $f \in \mathcal{H} \mathcal{M}^{!}(\lambda)$ as a series. For compactness of notation, we set, for each $j \in \mathbb{Z}, c_{m}^{(j)}=a_{m}^{(j)}+b_{m}^{(j)}$, where $a_{m}^{(j)}$ (resp. $b_{m}^{(j)}$ ) are taken to be 0 if $j$ or $m$ is outside the range of $j$ - or $m$-summation in (6) (resp. (7)). Then, by substituting the Fourier expansion of $f$ into (13), we deduce, for $w \neq-k_{0},-k_{0}+1, k_{0}+r+s-1, k_{0}+r+s$,

$$
L_{f}^{*}(w)=\sum_{j \in \mathbb{Z}} \sum_{m \neq 0} \frac{c_{m}^{(j)} \Gamma(w+j, 2 \pi m)}{(2 \pi m)^{w+j}}+i^{r-s} \sum_{j \in \mathbb{Z}} \sum_{m \neq 0} \frac{c_{m}^{(j)} \Gamma(s+r+j-w, 2 \pi m)}{(2 \pi m)^{s+r+j-w}}+P(w)
$$


where $P(w)$ denotes

$$
\begin{aligned}
\int_{0}^{1}\left(i^{r-s} f^{0}\left(\frac{i}{t}\right) t^{-r-s}-f^{0}(i t)\right) t^{w} \frac{\mathrm{d} t}{t}= & \frac{i^{r-s} a}{w-k_{0}-r-s}+\frac{i^{r-s} b}{w+k_{0}-1} \\
& -\frac{a}{w+k_{0}}-\frac{b}{w-k_{0}-r-s+1} .
\end{aligned}
$$

Because of Proposition 3.2 and the asymptotics $\Gamma(r, x) \sim e^{-x} x^{r-1}$ as $x \rightarrow \infty$, we see that this series converges absolutely for all $w \in \mathbb{C}$.

\subsubsection{Example: L-function of a weakly holomorphic modular form}

In the special case of a weakly holomorphic form, this formula coincides with the earlier definition of an $L$-function for such forms. Indeed, an $f \in M_{k}^{!}$can be considered as an element of $\mathcal{H M}^{!}(0) \cap \mathcal{M}_{k, 0}^{!}$with $k_{0}=1-k, a=b_{m}^{(j)}=0$ for all $j, m$ and $a_{m}^{(j)}=0$ for $j \neq 0$ or $m<m_{0}$ for some $m_{0} \in \mathbb{Z}$. Then (21) becomes

$$
L_{f}^{*}(w)=\sum_{m \geq m_{0}} \frac{a_{m}^{(0)} \Gamma(w, 2 \pi m)}{(2 \pi m)^{w}}+i^{k} \sum_{m \geq m_{0}} \frac{a_{m}^{(0)} \Gamma(k-w, 2 \pi m)}{(2 \pi m)^{k-w}}-b\left(\frac{1}{w}+\frac{i^{k}}{k-w}\right)
$$

which coincides with, say, (6.1) of [1].

\subsubsection{L-functions of modular iterated integrals of length 1}

Using Proposition 2.4, we can now express the $L$-function of a function $F$ in the broader class $\mathcal{M} \mathcal{I}_{1}^{!}$of modular iterated integrals of length 1 , in terms of the $L$-function in $\mathcal{H} \mathcal{M}^{!}(\lambda)$ for varying $\lambda$. Indeed, let $F$ be an element of $\mathcal{M} \mathcal{I}_{1}^{!}$of weights $r$, $s$. Then, for each $s \in \mathbb{C}$, we have

$$
L_{F}^{*}(s)=\sum_{k=0}^{\min (r, s)} L_{F_{k}}^{*}(s)
$$

where $F_{k}$ are elements of $\mathcal{H} \mathcal{M} !$ of weight $r, s$ such that $F=F_{0}+\cdots+F_{\min (r, s)}$ as in Proposition 2.4.

\subsection{L-functions in $\mathcal{M}_{r, s}$}

We now consider the case that $f$ is of polynomial growth at the cusps, i.e., $f \in \mathcal{M}_{r, s}$. Then, $\stackrel{\circ}{f}=0$ and $\tilde{f}=f-f^{0}$. Further, for $\Re(w) \gg 0$, the integral $\int_{0}^{\infty} \tilde{f}(i t) t^{w-1} \mathrm{~d} t$ converges, and therefore, we can make the change of variables $t \rightarrow 1 / t$ in the third integral of (13) to derive

$$
\begin{aligned}
L_{f}^{*}(w) & =\int_{1}^{\infty}\left(f(i t)-f^{0}(i t)\right) t^{w} \frac{\mathrm{d} t}{t}+i^{r-s} \int_{0}^{1}\left(f(i / t)-f^{0}(i / t)\right) t^{-r-s+w} \frac{\mathrm{d} t}{t} \\
& =\int_{0}^{\infty}\left(f(i t)-f^{0}(i t)\right) t^{w} \frac{\mathrm{d} t}{t}
\end{aligned}
$$

Here we used the transformation law for $f$ and the formula for the antiderivative of $f^{0}$.

This coincides with Brown's definition of $L$-functions of $f \in \mathcal{M}_{r, s}$ given in [3] (Sect. 9.4). There, up to a different normalisation, the $L$-function is actually defined, for $\operatorname{Re}(w) \gg 0$, by

$$
L_{f}^{*}(w)=\sum_{|j| \leq M}(2 \pi)^{-j-w} \Gamma(w+j) L_{f}^{(j)}(w+j)
$$


where, with the notation of (1),

$$
L_{f}^{(j)}(w):=\sum_{N \geq 1} \frac{1}{N^{w}}\left(\sum_{m+n=N} a_{m, n}^{(j)}\right) .
$$

The equivalence of this with our definition is established in the proof of Th. 9.7 of [3].

\subsubsection{L-functions in $\mathcal{H} \mathcal{M}^{!}(\lambda) \cap \mathcal{M}_{r, s}$}

When, in addition, $f \in \mathcal{M}_{r, s}$ is an eigen-function of the Laplacian, then by computing Mellin transforms as usual, $L_{f}^{*}(w)$ obtains a more familiar form which, however, is valid for $\Re(w) \gg 0$. Specifically, let $f \in \mathcal{H M}^{!}(\lambda) \cap \mathcal{M}_{r, s}$. By Lemma 2.3 , there is a $k_{0} \in \mathbb{Z}$ such that $f=f^{0}+f^{h}+f^{a}$ with

$$
\begin{aligned}
& f^{0}(z)=a y^{k_{0}}+b y^{1-r-s-k_{0}}, \quad f^{h}(z)=\sum_{j=k_{0}}^{-s} y^{j} \sum_{m>0} a_{m}^{(j)} q^{m} \text { and } \\
& f^{a}(z)=\sum_{j=k_{0}}^{-r} y^{j} \sum_{m>0} b_{m}^{(j)} \bar{q}^{m}
\end{aligned}
$$

$\left(a, b, a_{m}^{(j)}, b_{m}^{(j)} \in \mathbb{C}\right)$. Then for $\Re(w) \gg 0,(22)$ [or, directly, (13)] becomes

$$
L_{f}^{*}(w)=\sum_{j=-r-s}^{-s} \frac{\Gamma(j+w)}{(2 \pi)^{j+w}} \sum_{m>0} \frac{a_{m}^{(j)}}{m^{j+w}}+\sum_{j=-r-s}^{-r} \frac{\Gamma(j+w)}{(2 \pi)^{j+w}} \sum_{m>0} \frac{b_{m}^{(j)}}{m^{j+w}}
$$

\subsubsection{Example: $L$-function of the real-analytic Eisenstein series}

We can use the above representation of $L_{f}^{*}(s)$ and (8) to compute explicitly the $L$-function of the real-analytic Eisenstein series. For $\Re(w) \gg 0$, we have

$$
\begin{aligned}
L_{\mathcal{E}_{r, s}}^{*}(w)= & \frac{(2 \pi)^{r+s-w} \pi}{\Gamma(r+1) \zeta(r+s+1)}\left(\sum_{j=-r-s}^{-s} 2^{j} \Gamma(j+w) \alpha_{-j-\frac{r+s}{2}}^{+} \sum_{m>0} \frac{\sigma_{r+s+1}(m)}{m^{w+1}}\right. \\
& \left.+\sum_{j=-r-s}^{-r} 2^{j} \Gamma(j+w) \alpha_{-j-\frac{r+s}{2}}^{-} \sum_{m>0} \frac{\sigma_{r+s+1}(m)}{m^{w+1}}\right) \\
= & \frac{\zeta(w+1) \zeta(w-r-s)(2 \pi)^{r+s-w} \pi}{\Gamma(r+1) \zeta(r+s+1)} \\
& \times\left(\sum_{j=-r-s}^{-s} 2^{j} \Gamma(j+w) \alpha_{-j-\frac{r+s}{2}}^{+}+\sum_{j=-r-s}^{-r} 2^{j} \Gamma(j+w) \alpha_{-j-\frac{r+s}{2}}^{-}\right.
\end{aligned}
$$

(The sum $\sum_{m>0} \frac{\sigma_{r+s+1}(m)}{m^{w+1}}$ has been computed as in the case of $L$-functions of the usual Eisenstein series.) The last expression also gives the meromorphic continuation to the entire $w$-plane.

\section{Maass-Selberg forms}

In [14], the authors extend the classical theory of period polynomials of (holomorphic) cusp forms by assigning a period function to Maass cusp forms of weight 0 . Mühlenbruch [16] later generalised that to Maass cusp forms of real weight. One of the ways to define 
the period function, in both $[14,16]$, is based on a differential form called Maass-Selberg form. We recall its definition and some of its properties.

Let $f, g$ be smooth functions defined in an open subset $U$ of $\mathfrak{H} \cup \overline{\mathfrak{H}}$. For $z=x+i y$, set

$$
\{f, g\}^{+}:=f(z) g(z) \frac{\mathrm{d} z}{y} \quad \text { and } \quad\{f, g\}^{-}:=f(z) g(z) \frac{\mathrm{d} \bar{z}}{y} .
$$

Let $k \in 2 \mathbb{Z}$. The Maass-Selberg form is then defined by

$$
\eta_{k}(f, g):=\left\{\partial_{k / 2} f, g\right\}^{+}-\left\{f, \bar{\partial}_{k / 2} g\right\}^{-}
$$

(We normalise slightly differently from [16] because we use Brown's version of the Maass operators instead of the operators $E_{2 k}^{+}=2 \partial_{k}$ and $E_{2 k}^{-}=2 \bar{\partial}_{-k}$ used in [16].)

The next lemma summarises the properties of Maass-Selberg form we will be needing.

Lemma 4.1 (Lemma 39 of [16]) For each $\gamma \in \Gamma$, we have

1. If $\eta_{k}(f, g) \circ \gamma$ denotes the pull-back of the differential form $\eta_{k}(f, g)$ by the map $z \rightarrow \gamma z$ $(z \in U)$, then we have

$$
\eta_{k}(f, g) \circ \gamma=\eta_{k}\left(\left.f\right|_{k} \gamma,\left.g\right|_{-k} \gamma\right) .
$$

2. Suppose that, for some $\lambda \in \mathbb{R}$, we have $\Omega_{k} f=\lambda f$ and $\Omega_{-k} g=\lambda g$. Then $\eta_{k}(f, g)$ is closed.

\subsection{A Maass-Selberg form associated to modular iterated integrals of length one}

To define the Maass-Selberg form that we will associate to modular iterated integrals of length one, we need a function $R_{n, v}(n \in 2 \mathbb{Z}, v \in \mathbb{C})$ we now define. For $z \in \mathfrak{H} \cup \overline{\mathfrak{H}}$ and $\zeta \neq z, \bar{z}$, it is given by

$$
R_{n, v}(z, \zeta)=\left(\frac{\zeta-\bar{z}}{\zeta-z}\right)^{\frac{n}{2}}\left(\frac{\operatorname{Im} z}{(\zeta-z)(\zeta-\bar{z})}\right)^{\frac{1}{2}-v}
$$

For each $\zeta \in \mathbb{C}$, this gives a well-defined real-analytic function of $z$ if we restrict $z$ to the complement in $\mathfrak{H}$ of some path joining $\zeta$ and $\bar{\zeta}$ and then choose an appropriate branch for the implied logarithm. Likewise, for a suitable subset of $\overline{\mathfrak{H}}$.

For the specific values of $n, v$ we will use the function $R_{n, v}$, it can be defined for all $\zeta \in \mathbb{C}$ and $z \in \mathfrak{H}$. Specifically, for $n=s-r$ and $\mu_{k}=-k+(r+s+1) / 2$ with $k$ as in Proposition 2.4 we have,

$$
R_{n, \mu_{k}}(z, \zeta)=\left(\frac{\zeta-\bar{z}}{\zeta-z}\right)^{\frac{s-r}{2}}\left(\frac{\operatorname{Im} z}{(\zeta-z)(\zeta-\bar{z})}\right)^{k-\frac{r+s}{2}}=(\operatorname{Im} z)^{k-\frac{r+s}{2}}(\zeta-z)^{r-k}(\zeta-\bar{z})^{s-k} .
$$

Since $k \leq r, s$, this can be defined for all $z \in \mathfrak{H} \cup \overline{\mathfrak{H}}$.

The function $R_{n, \mu_{k}}$ satisfies

Lemma 4.2 Set $n=s-r$ and $\mu_{k}=-k+(r+s+1) / 2$ with $k \leq r, s$.

1. For each $\zeta \in \mathbb{C}$, we have

$$
\begin{aligned}
\bar{\partial}_{-\frac{n}{2}} R_{n, \mu_{k}}(\cdot, \zeta) & =\frac{1}{2}\left(1-2 \mu_{k}-n\right) R_{n-2, \mu_{k}}(\cdot, \zeta) \\
\Omega_{n} R_{n, \mu_{k}}(\cdot, \zeta) & =\left(\frac{1}{4}-\mu_{k}^{2}\right) R_{n, \mu_{k}}(\cdot, \zeta) .
\end{aligned}
$$


2. For each $\gamma \in \Gamma$ and $\zeta \in \mathfrak{H}$, we have

$$
R_{n, \mu_{k}}(\gamma z, \gamma \zeta)=j(\gamma, \zeta)^{1-2 \mu_{k}}\left(\frac{j(\gamma, z)}{\mid j(\gamma, z \mid}\right)^{n} R_{n, \mu_{k}}(z, \zeta)
$$

Proof This is essentially Prop. 36 of [16] but there it is proved with the restriction that $\zeta \in \mathbb{R}$ and $j(\gamma, \zeta)>0$ due to the more general $\mu$ and $n$ to which the proposition applies.

We now associate to $F_{k}$ Maass-Selberg forms which will be the basis for our construction of the period function for all functions in $\mathcal{M I}_{1}^{!}$.

Proposition 4.3 Let $k \in\{0, \ldots, \min (r, s)\}$ and $\mu_{k}=-k+(r+s+1) / 2$. For each $\zeta \in \mathbb{C}$, the forms $\eta_{r-s}\left(y^{\frac{r+s}{2}}\left(\tilde{F}_{k}+a y^{k-r-s}\right), R_{s-r, \mu_{k}}(\cdot, \zeta)\right), \quad \eta_{r-s}\left(y^{\frac{r+s}{2}} \stackrel{\circ}{F}_{k}, R_{s-r, \mu_{k}}(\cdot, \zeta)\right)$ and $\eta_{r-s}\left(y^{\frac{r+s}{2}}\left(b y^{1-k}\right), R_{s-r, \mu_{k}}(\cdot, \zeta)\right)$ are closed.

Proof By Proposition 2.5, $y^{\frac{r+s}{2}}\left(\tilde{F}_{k}+a y^{k-r-s}\right), y^{\frac{r+s}{2}} \stackrel{\circ}{F}_{k}, y^{\frac{r+s}{2}}\left(b y^{1-k}\right)$, are eigenfunctions of $\Omega_{r-s}$ and, by Lemma $4.2, R_{s-r, \mu_{k}}(\cdot, \zeta)$ is an eigenfunction of $\Omega_{s-r}$. They all have eigenvalue $\frac{1}{4}-\mu_{k}^{2}$. Therefore, with Lemma 4.1 we deduce the assertion.

\section{Cocycles associated to modular iterated integrals of length one}

We briefly recall the basic cohomological formalism we will need. Let $M$ be a right $\Gamma$ module. If, for a non-negative integer $i, C^{i}(\Gamma, M)=\left\{s: \Gamma^{i} \rightarrow M\right\}$ denotes the space of $i$-cochains for $\Gamma$ with coefficients in $M$, we define the differential $d^{i}: C^{i}(\Gamma, M) \rightarrow$ $C^{i+1}(\Gamma, M)$ by

$$
\begin{aligned}
& \left(d^{i} \sigma\right)\left(g_{1}, \ldots, g_{i+1}\right) \\
& \quad:=\sigma\left(g_{2}, \ldots, g_{i+1}\right) . g_{1}+\sum_{j=1}^{i}(-1)^{j} \sigma\left(g_{1}, \ldots, g_{j+1} g_{j}, \ldots, g_{i+1}\right)+(-1)^{i+1} \sigma\left(g_{1}, \ldots, g_{i}\right) .
\end{aligned}
$$

Then, we define $Z^{i}(\Gamma, M):=\operatorname{Ker}\left(d^{i}\right)$ (group of $i$-cocyles), $B^{i}(\Gamma, M):=\operatorname{Im}\left(d^{i-1}\right)$ (group of $i$-coboundaries) and $H^{i}(\Gamma, M):=Z^{i}(\Gamma, M) / B^{i}(\Gamma, M)(i$-th cohomology group of $M)$.

In Eichler cohomology, the module $M$ is the space $P_{m}(K)$ of polynomial functions of degree $\leq m$ and coefficients in a field $K$, acted upon by $\underset{-m, 0}{\|}$. An important theorem is the Eichler Shimura isomorphism

$$
\phi: \overline{S_{k}} \oplus M_{k} \stackrel{\sim}{\rightarrow} H^{1}\left(\Gamma, P_{k-2}(\mathbb{C})\right)
$$

where $M_{k}$ (resp. $S_{k}$ ) is the space of classical holomorphic modular (resp. cusp) forms of weight $k$ for $\Gamma$. The isomorphism $\phi$ is induced by the assignment of $f \in M_{k}$ to the map $\phi(f): \Gamma \rightarrow P_{k-2}(\mathbb{C})$ such that

$$
\phi(f)(\gamma)=\int_{i}^{\gamma^{-1} i} f(w)(w-z)^{k-2} \mathrm{~d} w \quad \text { for } \gamma \in \Gamma .
$$

We will now associate to the $F_{k}$ 's of the last section a 1-cocycle in the $\Gamma$-module $P_{r+s-2 k}(\mathbb{C})$. We define it as the coboundary of a 0 -cochain in a larger module than $P_{-2 k+r+s}(\mathbb{C})$. The construction follows the definition of the "integral at a tangential base point at infinity" of [6], (Sect. 4).

For convenience of notation, we set $\eta_{r-s}(g ; \zeta):=\eta_{r-s}\left(y^{\frac{r+s}{2}} g, R_{s-r, \mu_{k}}(\cdot, \zeta)\right)$ 
Proposition 5.1 Let $k \in\{0, \ldots, \min (r, s)\}$ and $\mu_{k}=-k+(r+s+1) / 2$. The function $v_{k}: \mathfrak{H} \rightarrow \mathbb{C}$ given by

$$
v_{k}(\zeta):=\int_{\zeta}^{i \infty} \eta_{r-s}\left(\tilde{F}_{k}+a y^{k-r-s} ; \zeta\right)+\int_{\zeta}^{0} \eta_{r-s}\left(b y^{1-k} ; \zeta\right)+\int_{\zeta}^{-i \infty} \eta_{r-s}\left(\stackrel{\circ}{F}_{k} ; \zeta\right),
$$

where the line of integration in the last integral includes the origin is well-defined. The differential forms to be integrated in $v_{k}$ can be written more explicitly in the form

$$
y^{k-1} \partial_{r} f(z)(\zeta-\bar{z})^{s-k}(\zeta-z)^{r-k} \mathrm{~d} z+(s-k) y^{k-1} f(z)(\zeta-\bar{z})^{s-k-1}(\zeta-z)^{r-k+1} \mathrm{~d} \bar{z}
$$

for each smooth function $f: \mathfrak{H} \cup \overline{\mathfrak{H}} \rightarrow \mathbb{C}$.

Proof We first show the second assertion. With the definition of $\eta_{r-s}$ and Lemma 4.2, we have:

$$
\begin{aligned}
\eta_{r-s}\left(y^{\frac{r+s}{2}} f, R_{s-r, \mu_{k}}(\cdot, \zeta)\right)= & \left(2 i y \frac{\partial}{\partial z}\left(y^{\frac{r+s}{2}} f(z)\right)+\frac{r-s}{2} y^{\frac{r+s}{2}} f(z)\right) R_{s-r, \mu_{k}}(\cdot, \zeta) \frac{\mathrm{d} z}{y} \\
& -y^{\frac{r+s}{2}} f(z)\left(\frac{1-2 \mu_{k}-s+r}{2}\right) R_{s-r-2, \mu_{k}}(\cdot, \zeta) \frac{\mathrm{d} \bar{z}}{y} \\
= & \left(r y^{\frac{r+s}{2}} f(z)+2 i y^{\frac{r+s}{2}+1} \frac{\partial f}{\partial z}(z)\right) R_{s-r, \mu_{k}}(\cdot, \zeta) \frac{\mathrm{d} z}{y}-y^{\frac{r+s}{2}} f(z) \\
& \times\left(\frac{1-2 \mu_{k}-s+r}{2}\right) R_{s-r-2, \mu_{k}}(\cdot, \zeta) \frac{\mathrm{d} \bar{z}}{y}
\end{aligned}
$$

Substituting the value for $\mu_{k}$, we get (27). From this, we deduce that, if $f$ and $\partial f / \partial z$ decay exponentially as $y \rightarrow \infty$, the same holds for $\eta_{r-s}\left(y^{\frac{r+s}{2}} f, R_{s-r, \mu_{k}}(\cdot, \zeta)\right)$. This condition holds for $f=\tilde{F}_{k}$. It also holds for $f=\stackrel{\circ}{F}_{k}$ as $y \rightarrow-\infty$.

The term corresponding to $a y^{k-r-s}$ in the first integral is $O\left(y^{-2}\right)$ as $y \rightarrow \infty$, which assures convergence. (Note that each of the two summands in (27) individually has a term of order $y^{-1}$ but they cancel each other out on the upper imaginary axis).

Since it is clear that the second integral in the definition of $v_{f}$ is convergent too, we deduce that, for each $\zeta \in \mathbb{C}$, all integrals are convergent.

Further, by Proposition 4.3, $\eta_{r-s}\left(\tilde{F}_{k}+a y^{k-r-s} ; \zeta\right), \eta_{r-s}\left(b y^{1-k} ; \zeta\right)$ and $\eta_{r-s}\left(\stackrel{\circ}{F}_{k}, \zeta\right)$ are closed in $\mathfrak{H}$. The last form is also closed in $\overline{\mathfrak{H}}$. Indeed, for each fixed $\zeta \in \mathbb{C}$, by (27), we have that $d\left(\eta_{r-s}\left(\stackrel{\circ}{F}_{k}, \zeta\right)\right)=P\left[e^{-2 \pi i z}\right] \mathrm{d} z \wedge d \bar{z}$, where $P$ is a polynomial in $e^{-2 \pi i z}$ whose coefficients are polynomials in $z$, $\bar{z}$. Since $\eta_{r-s}\left(\stackrel{\circ}{F}_{k}, \zeta\right)$ is closed in $\mathfrak{H}$, each of those polynomials are identically zero in $\mathfrak{H}$, and therefore, they vanish in $\overline{\mathfrak{H}}$ too.

We can now define the 1-cocyle $\sigma_{k}$ on $\Gamma$ as

$$
\sigma_{k}=d^{0} v_{k}
$$

We will show that, although $v_{k}$ does not belong to $P_{r+s-2 k}(\mathbb{C})$, its differential does and, in fact, it belongs to a cohomology class analogous to that of (26) in the classical Eichler cohomology.

Proposition 5.2 Let $F \in \mathcal{M I}_{1}^{!} \cap \mathcal{M}_{r, s}$. For $k \in\{0, \ldots, \min (r, s)\}$, let $F_{k}$ be the $k$-th term in the decomposition of $F$ in eigenfunctions of $\Delta_{r, s}$ as in Proposition 2.4. Then

1. The map $\sigma_{k}$ induces a 1-cocycle in $P_{r+s-2 k}(\mathbb{C})$. 
2. Let $\tilde{\sigma}_{k}: \Gamma \rightarrow P_{r+s-2 k}(\mathbb{C})$ be the map given by

$$
\tilde{\sigma}_{k}(\gamma)(\zeta)=\int_{i}^{\gamma^{-1} i} \eta_{r-s}\left(y^{\frac{r+s}{2}} F_{k}, R_{s-r, \mu_{k}}(\cdot, \zeta)\right)
$$

This gives a 1-cocycle which belongs to the same cohomology class as $\sigma_{k}$.

Proof We occasionally use again the abbreviation $\eta_{r-s}(g ; \zeta):=\eta_{r-s}\left(y^{\frac{r+s}{2}} g, R_{s-r, \mu_{k}}(\cdot, \zeta)\right)$.

Since $\eta_{r-s}(g ; \zeta)$ are closed for $g=\tilde{F}_{k}, a y^{k-r-s}, b y^{1-k}$ and $\stackrel{\circ}{F}_{k}$, we have

$$
\begin{aligned}
v_{k}(\zeta)= & \int_{\zeta}^{i} \eta_{r-s}\left(F_{k}-b y^{1-k}-\stackrel{\circ}{F}_{k} ; \zeta\right)+\int_{i}^{i \infty} \eta_{r-s}\left(\tilde{F}_{k}+a y^{k-r-s} ; \zeta\right) \\
& +\int_{\zeta}^{0} \eta_{r-s}\left(b y^{1-k} ; \zeta\right)+\int_{\zeta}^{-i \infty} \eta_{r-s}\left(\stackrel{\circ}{F}_{k} ; \zeta\right) \\
= & \int_{\zeta}^{i} \eta_{r-s}\left(F_{k} ; \zeta\right)+\int_{i}^{i \infty} \eta_{r-s}\left(\tilde{F}_{k}+a y^{k-r-s} ; \zeta\right)+\int_{i}^{0} \eta_{r-s}\left(b y^{1-k} ; \zeta\right) \\
& +\int_{i}^{-i \infty} \eta_{r-s}\left(\stackrel{\circ}{F}_{k} ; \zeta\right)
\end{aligned}
$$

where the last integral is also taken to be over a path that includes the origin. By (27), the last three terms of $(28)$ are clearly in $P_{r+s-2 k}(\mathbb{C})$. (However, note that to reach this conclusion, we first fix a specific path of integration and only then expand the integrand in $\zeta$. If we first expanded, there would be no guarantee $a$ priori that the resulting differentials are closed). Thus, the image of those integrals under the action by $\|_{2 k-r-s, 0}(\gamma-1)$ is in $P_{r+s-2 k}(\mathbb{C})$ too.

To show that $\int_{\zeta}^{i} \eta_{r-s}\left(F_{k} ; \zeta\right) \|_{2 k-r-s, 0}(\gamma-1) \in P_{r+s-2 k}(\mathbb{C})$, we observe that, by Lemma 4.1 (1), we have, for each $\gamma \in \Gamma$,

$$
\eta_{r-s}\left(y^{\frac{r+s}{2}} F_{k}, R_{s-r, \mu_{k}}(\cdot, \gamma \zeta)\right) \circ \gamma=\eta_{r-s}\left(\left.\left(y^{\frac{r+s}{2}} F_{k}\right)\right|_{r-s} \gamma,\left.R_{s-r, \mu_{k}}(\cdot, \gamma \zeta)\right|_{s-r} \gamma\right)
$$

where the action $\left.\right|_{s-r}$ refers to the implied variable $z$. By Proposition 2.5 and Lemma 4.2(2), the last term equals $\eta_{r-s}\left(y^{\frac{r+s}{2}} F_{k}, R_{s-r, \mu_{k}}(\cdot, \zeta)\right) j(\gamma, \zeta)^{1-2 \mu_{k}}$. Therefore,

$$
\begin{aligned}
& \left(\int_{\zeta}^{i} \eta_{r-s}\left(y^{\frac{r+s}{2}} F_{k}, R_{s-r, \mu_{k}}(\cdot, \zeta)\right)\right) \|_{1-2 \mu_{k}, 0} \gamma=\left(\int_{\gamma \zeta}^{i} \eta_{r-s}\left(y^{\frac{r+s}{2}} F_{k}, R_{s-r, \mu_{k}}(\cdot, \gamma \zeta)\right)\right) j(\gamma, \zeta)^{2 \mu_{k}-1} \\
& =\left(\int_{\zeta}^{\gamma^{-1} i} \eta_{r-s}\left(y^{\frac{r+s}{2}} F_{k}, R_{s-r, \mu_{k}}(\cdot, \gamma \zeta)\right) \circ \gamma\right) j(\gamma, \zeta)^{2 \mu_{k}-1}=\int_{\zeta}^{\gamma^{-1} i} \eta_{r-s}\left(y^{\frac{r+s}{2}} F_{k}, R_{s-r, \mu_{k}}(\cdot, \zeta)\right)
\end{aligned}
$$

This implies that

$$
\left(\int_{\zeta}^{i} \eta_{r-s}\left(y^{\frac{r+s}{2}} F_{k}, R_{s-r, \mu_{k}}(\cdot, \zeta)\right)\right) \|_{1-2 \mu_{k}, 0}(\gamma-1)=\int_{i}^{\gamma^{-1} i} \eta_{r-s}\left(y^{\frac{r+s}{2}} F_{k}, R_{s-r, \mu_{k}}(\cdot, \zeta)\right)
$$

which is in $P_{r+s-2 k}(\mathbb{C})$. Therefore, $\sigma_{k}(\gamma) \in P_{r+s-2 k}(\mathbb{C})$ and since, further, $\sigma_{k}$ is given as the differential of a 0 -cochain, we deduce 1 .

To derive 2., we see with (28) and (29) that, for all $\gamma \in \Gamma$ and $\zeta \in \mathfrak{H}$,

$$
\begin{aligned}
& \sigma_{k}(\gamma)(\zeta)=\tilde{\sigma}_{k}(\gamma)(\zeta) \\
& \quad+\left(\int_{i}^{i \infty} \eta_{r-s}\left(\tilde{F}_{k}+a y^{k-r-s} ; \zeta\right)+\int_{i}^{-i \infty} \eta_{r-s}\left(\stackrel{\circ}{F}_{k} ; \zeta\right)+\int_{i}^{0} \eta_{r-s}\left(b y^{1-k} ; \zeta\right)\right) \|_{2 k-r-s, 0}(\gamma-1) .
\end{aligned}
$$


Since the last integrals belong to $P_{r+s-2 k}(\mathbb{C})$, we deduce, on the one hand, that $\tilde{\sigma}_{k}$ is a 1-cocycle with coefficients in $P_{r+s-2 k}(\mathbb{C})$ and, on the other, that $\sigma_{k}$ and $\tilde{\sigma}_{k}$ differ by a 1 -coboundary in $P_{r+s-2 k}(\mathbb{C})$. Therefore, they belong to the same cohomology class.

As in the case of the classical period polynomial, the value of this cocycle at the involution $S$ encapsulates the critical values of the $L$-functions of $F_{k}$. However, in the general case, its leading and constant terms must be "truncated".

Theorem 5.3 Assume that $r \equiv s \bmod 4$. Then,

$$
\sigma_{k}(S)(\zeta)-\sigma_{k}^{0}(S)(\zeta)=i \sum_{l=1}^{s+r-2 k-1}\left(\sum_{n=0}^{l} \alpha_{n, l}\right) L_{F_{k}}^{*}(k+l) \zeta^{l}
$$

where $\sigma_{k}^{0}(S)(\zeta)$ denotes the sum of the leading and constant term of $\sigma_{k}(\zeta)$ and

$$
\alpha_{n, l}=i^{-l-2 n}\left((r-k)\left(\begin{array}{c}
s-k+1 \\
l-n
\end{array}\right)\left(\begin{array}{c}
r-k+1 \\
n
\end{array}\right)-(s-k)\left(\begin{array}{c}
s-k-1 \\
l-n
\end{array}\right)\left(\begin{array}{c}
r-k+1 \\
n
\end{array}\right)\right)
$$

Proof We notice, with (30) that $\sigma_{k}(S)(\zeta)$ equals

$$
\left.\left(\int_{i}^{i \infty} \eta_{r-s}\left(\tilde{F}_{k}+a y^{k-r-s} ; \zeta\right)+\int_{i}^{-i \infty} \eta_{r-s}\left(\stackrel{\circ}{F}_{k} ; \zeta\right)+\int_{i}^{0} \eta_{r-s}\left(b y^{1-k} ; \zeta\right)\right)\right) \|_{2 k-r-s, 0}(S-1)
$$

Now, each smooth function $h: \mathfrak{H} \cup \overline{\mathfrak{H}} \rightarrow \mathbb{C}$, we deduce from (27) that

$$
\begin{aligned}
\eta_{r-s}(h ; \zeta)= & 2 i \frac{\partial}{\partial z}\left(y^{k} h(z)(\zeta-\bar{z})^{s-k}(\zeta-z)^{r-k}\right)+(r-k) h(z)(\zeta-\bar{z})^{s-k+1}(\zeta-z)^{r-k-1} \\
& -(s-k) h(z)(\zeta-\bar{z})^{s-k-1}(\zeta-z)^{r-k+1}
\end{aligned}
$$

We further notice that $\eta_{r-s}\left(y^{k-r-s} ; 0\right)=0$. Therefore, $\eta_{r-s}\left(y^{k-r-s} ; \zeta\right)=\eta_{r-s}\left(y^{k-r-s} ; \zeta\right)-$ $\eta_{r-s}\left(y^{k-r-s} ; 0\right)$ equals

$$
\begin{aligned}
& 2 i \frac{\partial}{\partial z}\left(y^{k} \cdot y^{k-r-s}\left((\zeta-\bar{z})^{s-k}(\zeta-z)^{r-k}-\bar{z}^{s-k} z^{r-k}\right)\right) \\
& \quad+(r-k) \cdot y^{k-r-s}\left((\zeta-\bar{z})^{s-k+1}(\zeta-z)^{r-k-1}-\bar{z}^{s-k+1} z^{r-k-1}\right) \\
& \quad-(s-k) \cdot y^{k-r-s}\left((\zeta-\bar{z})^{s-k-1}(\zeta-z)^{r-k+1}-\bar{z}^{s-k-1} z^{r-k+1}\right) .
\end{aligned}
$$

Each of the polynomials in $\zeta$ have degree $\leq s+r-2 k-1$. Hence, the integral $\int_{i}^{i \infty} \eta_{r-s}\left(y^{k-r-s} ; \zeta\right)$ converges and, by integrating along the positive imaginary axis, we deduce that it equals

$$
\begin{aligned}
& i \int_{1}^{\infty} y^{2 k-r-s-1}\left[(r-k)\left((\zeta+i y)^{s-k+1}(\zeta-i y)^{r-k-1}-(i y)^{s-k+1}(-i y)^{r-k-1}\right)\right. \\
& \left.\quad-(s-k)\left((\zeta+i y)^{s-k-1}(\zeta-i y)^{r-k+1}-(i y)^{s-k+1}(-i y)^{r-k-1}\right)\right] \mathrm{d} y \\
& \quad-2 i\left((\zeta+i)^{s-k}(\zeta-i)^{r-k}-1\right)
\end{aligned}
$$

Equation (32) can be used directly for $h=\tilde{F}_{k}, \stackrel{\circ}{F}_{k}, b y^{1-k}$, to yield

$$
\begin{gathered}
\int_{i}^{i \infty} \eta_{r-s}\left(y^{\frac{r+s}{2}} h, R_{s-r, \mu_{k}}(\cdot, \zeta)\right)=(r-k) i \int_{1}^{\infty} t^{k-1} h(i t)(\zeta+i t)^{s-k+1}(\zeta-i t)^{r-k-1} \mathrm{~d} t \\
-(s-k) i \int_{1}^{\infty} t^{k-1} h(i t)(\zeta+i t)^{s-k-1}(\zeta-i t)^{r-k+1} \mathrm{~d} t-2 i h(i)(\zeta+i)^{s-k}(\zeta-i)^{r-k}
\end{gathered}
$$


Therefore, with (31) and (34) we deduce that $\sigma_{k}(S)(\zeta)-\sigma_{k}^{0}(S)(\zeta)$ equals

$$
\begin{aligned}
& i \int_{1}^{\infty} t^{k-1}\left(\tilde{F}_{k}(i t)+a t^{k-r-s}\right)\left(R(t, \zeta)-R_{0}(t, \zeta)\right) \mathrm{d} t \\
& \quad+i \int_{1}^{-\infty} t^{k-1} \stackrel{\circ}{F}_{k}(i t)\left(R(t, \zeta)-R_{0}(t, \zeta)\right) \mathrm{d} t \\
& \quad+i \int_{1}^{0} t^{k-1}\left(b t^{1-k}\right)\left(R(t, \zeta)-R_{0}(t, \zeta)\right) \mathrm{d} t
\end{aligned}
$$

where

$$
\begin{aligned}
R(t, \zeta):= & (r-k)\left((1-i t \zeta)^{s-k+1}(1+i t \zeta)^{r-k-1}-(\zeta+i t)^{s-k+1}(\zeta-i t)^{r-k-1}\right) \\
& -(s-k)\left((1-i t \zeta)^{s-k-1}(1+i t \zeta)^{r-k+1}-(\zeta+i t)^{s-k-1}(\zeta-i t)^{r-k+1}\right)
\end{aligned}
$$

and $R_{0}(t, \zeta)$ is the sum of the constant and the leading term of the expansion of $R(t, \zeta)$ in $\zeta$. (The terms of order between 1 and $s+r-2 k-1$ coming from $2 i h(i)(\zeta+i)^{s-k}(\zeta-i)^{r-k}$ and $2 i\left((\zeta+i)^{s-k}(\zeta-i)^{r-k}-1\right)$ cancel because $s \equiv r \bmod 4$. $)$

Using the binomial expansion, we see that the integral in the RHS of (35) equals, in the notation of the statement of the proposition:

$$
\begin{aligned}
& \sum_{l=1}^{s+r-2 k-1}\left(\sum_{n=0}^{l} \alpha_{n, l}\right)\left(\int_{1}^{\infty}\left(\tilde{F}_{k}(i t)+a t^{k-r-s}\right)\left(t^{k+l}+i^{s-r} t^{s+r-k-l}\right) \frac{\mathrm{d} t}{t}\right. \\
& \left.+\int_{1}^{-\infty} \stackrel{\circ}{F_{k}}(i t)\left(t^{k+l}+i^{s-r} t^{s+r-k-l}\right) \frac{\mathrm{d} t}{t}+\int_{1}^{0} b t^{1-k}\left(t^{k+l}+i^{s-r} t^{s+r-k-l}\right) \frac{\mathrm{d} t}{t}\right) \zeta^{l} .
\end{aligned}
$$

With the definition of $L_{F_{k}}^{*}$, we deduce the proposition.

From Proposition 5.2, we obtain a map from the space of modular iterated integrals of length one to a direct sum of copies of the space of classical modular (resp. cusp) forms.

Theorem 5.4 The maps $\sigma_{k}(k \in\{0, \ldots, \min (r, s)\})$ defined in Proposition 5.2 induce a map

$$
\mathcal{M I}_{1}^{!} \cap \mathcal{M}_{r, s}^{!} \rightarrow \bigoplus_{k=0}^{\min (r, s)} H^{1}\left(\Gamma, P_{r+s-2 k}(\mathbb{C})\right) \cong \bigoplus_{k=0}^{\min (r, s)}\left(\bar{S}_{r+s-2 k+2} \oplus M_{r+s-2 k+2}\right)
$$

Proof Proposition 5.2 induces a map sending each $F \in \mathcal{M I}_{1}^{!} \cap \mathcal{M}_{r, s}^{!}$to

$$
\left(\left[\sigma_{0}\right], \ldots\left[\sigma_{\min (r, s)}\right]\right) \in \bigoplus_{k=0}^{\min (r, s)} H^{1}\left(\Gamma, P_{r+s-2 k}(\mathbb{C})\right)
$$

Here $\left[\sigma_{k}\right]$ stands for the cohomology class of the 1-cocycle defined in that proposition. The last isomorphism of the theorem follows from the Eichler-Shimura isomorphism [see (25)].

Corollary 5.5 Let $F$ be a modular iterated integral of length one and weights $(r, s)$ and let $F=F_{0}+\cdots+F_{\min (r, s)}$ be its decomposition into eigenfunctions of the Laplacian. Then, for 
each $k \in\{0, \ldots, \min (r, s)\}$, there is a $P_{k}(\zeta) \in P_{r+s-2 k}(\mathbb{C})$ and unique $f_{k} \in S_{r+s-2 k+2}, g_{k} \in$ $M_{r+s-2 k+2}$ such that, for all $\gamma \in \Gamma$,

$$
\begin{aligned}
\int_{i}^{\gamma^{-1} i} \eta_{r-s}\left(y^{\frac{r+s}{2}} F_{k}, R_{s-r, \mu_{k}}(\cdot, \zeta)\right)= & \int_{i}^{\gamma^{-1} i} f(z)(z-\zeta)^{r+s-2 k} \mathrm{~d} z \\
& +\int_{i}^{\gamma^{-1} i} g(z)(z-\bar{\zeta})^{r+s-2 k} \mathrm{~d} z \\
& +P_{k} \|_{2 k-r-s, 0}(\gamma-1) .
\end{aligned}
$$

\section{An application to algebraicity}

In [7] an Eichler-Shimura isomorphism for weakly holomorphic modular forms is proved, which respects rational structures. As a proof of concept for the "correctness" of our definition of the $L$-function in Sect. 3, we will use the results of [7] to show an analogue of Manin's Periods Theorem [15] for weakly holomorphic forms. It should be mentioned that K. Bringmann has shown us an alternative way, based on results of [2], to establish a statement implying the same result.

Before stating and proving our result, we first summarise the setup of [7] and then show that it is compatible with the explicit expressions for the cocycles of the last section.

Let $M_{k, \mathbb{Q}}^{!}$, resp. $S_{k, \mathbb{Q}}^{!}$denote the $\mathbb{Q}$-vector space of weight $k$ weakly holomorphic modular, resp. cusp, forms for $\Gamma=\mathrm{SL}_{2}(\mathbb{Z})$, with rational Fourier coefficients. Consider the differential operator $D=\frac{1}{2 \pi i} \frac{\mathrm{d}}{\mathrm{d} z}$. In [12], it is shown that, although there are generally no Hecke eigenforms in $S_{k, \mathbb{Q}}^{!}$, there are well-defined operators on $M_{k, \mathbb{Q}}^{!} / D^{k-1} M_{2-k, \mathbb{Q}}^{!}$induced by the standard Hecke operators and, within that space, there are Hecke invariant classes. With this terminology and notation, we have

Theorem 6.1 (Cor. 1.3 of [7]) The map $\phi$ assigning to $f \in M_{k}^{!}$the function (26) induces a Hecke invariant isomorphism

$$
[\phi]: M_{k, \mathbb{Q}}^{!} / D^{k-1} M_{2-k, \mathbb{Q}}^{!} \otimes_{\mathbb{Q}} \mathbb{C} \stackrel{\sim}{\rightarrow} H^{1}\left(\Gamma, P_{k-2}(\mathbb{Q})\right) \otimes_{\mathbb{Q}} \mathbb{C}
$$

The image of $S_{k, \mathbb{Q}}^{!} / D^{k-1} M_{2-k, \mathbb{Q}}^{!} \otimes_{\mathbb{Q}} \mathbb{C}$ is the parabolic cohomology group defined by:

$$
H_{\mathrm{par}}^{1}\left(\Gamma, P_{k-2}(\mathbb{C})\right):=Z_{\mathrm{par}}^{1}\left(\Gamma, P_{k-2}(\mathbb{C})\right) / B_{\mathrm{par}}^{1}\left(\Gamma, P_{k-2}(\mathbb{C})\right)
$$

where $Z_{\text {par }}^{1}\left(\Gamma, P_{k-2}(\mathbb{C})\right):=\left\{\psi \in Z^{1}\left(\Gamma, P_{k-2}(\mathbb{C})\right) ; \psi(T)=0\right\}$ is the space of parabolic cocycles and $B_{\text {par }}^{1}\left(\Gamma, P_{k-2}(\mathbb{C})\right):=\left\{\psi \in B^{1}\left(\Gamma, P_{k-2}(\mathbb{C})\right) ; \psi(T)=0\right\}$ the space of parabolic coboundaries. These spaces are defined over $\mathbb{Q}$ and $B_{\mathrm{par}}^{1}\left(\Gamma, P_{k-2}(\mathbb{C})\right)$ is generated by $\psi_{0}$ such that

$$
\psi_{0}(\gamma)=1 \underset{2-k, 0}{\|}(\gamma-1) .
$$

Further, let $F_{\infty}$ be the "real Frobenius" induced by the map sending $\sigma \in Z_{\mathrm{par}}^{1}\left(\Gamma, P_{k-2}(\mathbb{C})\right)$ to $F_{\infty} \sigma \in Z_{\mathrm{par}}^{1}\left(\Gamma, P_{k-2}(\mathbb{C})\right)$ such that

$$
F_{\infty} \sigma\left(\begin{array}{ll}
a & b \\
c & d
\end{array}\right)(z)=\sigma\left(\begin{array}{cc}
a & -b \\
-c & d
\end{array}\right)(-z) .
$$

Then $H_{\text {par }}^{1}\left(\Gamma, P_{k-2}(\mathbb{C})\right)$ is decomposed canonically into $F_{\infty}$-eigenspaces:

$$
H_{\mathrm{par}}^{1}\left(\Gamma, P_{k-2}(\mathbb{C})\right)=H_{\mathrm{par}}^{1,+}\left(\Gamma, P_{k-2}(\mathbb{C})\right) \oplus H_{\mathrm{par}}^{1,-}\left(\Gamma, P_{k-2}(\mathbb{C})\right) .
$$


Each class of $H_{\text {par }}^{1,+}\left(\Gamma, P_{k-2}(\mathbb{C})\right)\left(\right.$ resp. $H_{\text {par }}^{1,-}\left(\Gamma, P_{k-2}(\mathbb{C})\right)$ is represented by a cocycle $\sigma$ such that $\sigma(S)$ is an even (resp. odd) polynomial.

Let now $\phi^{\prime}$ be the map assigning to each $f \in S_{k, \mathbb{Q}}^{!}$the cocyle $\sigma_{f}^{\prime}=d^{0} v_{f}^{\prime}$ where $v_{f}^{\prime}: \mathfrak{H} \rightarrow$ $P_{k-2}(\mathbb{C})$ is given by

$$
v_{f}^{\prime}(z):=\int_{i \infty}^{z} \tilde{f}(w)(w-z)^{k-2} \mathrm{~d} w+\int_{-i \infty}^{z} \tilde{f}(w)(w-z)^{k-2} \mathrm{~d} w,
$$

$\tilde{f}, \stackrel{\circ}{f}$ defined by (2), (4), respectively.

We will prove the proposition

Proposition 6.2 The map $\phi^{\prime}$ induces a map $\left[\phi^{\prime}\right]$ from the space $S_{k}^{!} / D^{k-1} M_{2-k}^{!} \otimes_{\mathbb{Q}} \mathbb{C}$ to $H_{\text {par }}^{1}\left(\Gamma, P_{k-2}(\mathbb{Q})\right) \otimes_{\mathbb{Q}} \mathbb{C}$. The resulting diagram

$$
\begin{gathered}
M_{k}^{!} / D^{k-1} M_{2-k}^{!} \otimes_{\mathbb{Q}} \mathbb{C} \stackrel{[\phi]}{\longrightarrow} H^{1}\left(\Gamma, P_{k-2}(\mathbb{Q})\right) \otimes_{\mathbb{Q}} \mathbb{C} \\
i \uparrow \\
S_{k}^{!} / D^{k-1} M_{2-k}^{!} \otimes_{\mathbb{Q}} \mathbb{C} \stackrel{\left[\phi^{\prime}\right]}{\longrightarrow} H_{p a r}^{1}\left(\Gamma, P_{k-2}(\mathbb{Q})\right) \otimes_{\mathbb{Q}} \mathbb{C}
\end{gathered}
$$

(where $i, j$ are natural injections and $\phi$ is as in Theorem 6.1) is commutative.

Proof Let $f \in S_{k, \mathbb{Q}}^{!}$. Then since both $\tilde{f}$ and $\stackrel{\circ}{f}$ are periodic with period 1 , it is easy to see that $\sigma_{f}^{\prime}(T)=v_{f}^{\prime} \|(T-1)(z)=v_{f}^{\prime}(z+1)-v_{f}^{\prime}(z)=0$. In addition, $\sigma_{f}^{\prime}$ is a cocycle by construction and therefore it is a parabolic cocycle. This proves the first assertion.

Now,

$$
v_{f}^{\prime}(z)=\int_{i}^{z} f(w)(w-z)^{k-2} \mathrm{~d} w+\int_{i \infty}^{i} \tilde{f}(w)(z-w)^{k-2} \mathrm{~d} w+\int_{-i \infty}^{i} \dot{f}(w)(z-w)^{k-2} \mathrm{~d} w
$$

and thus

$$
\begin{aligned}
\sigma_{f}^{\prime}(\gamma)= & \int_{\gamma^{-1} i}^{i} f(w)(\cdot-w)^{k-2} \mathrm{~d} w \\
& +\left[\int_{i \infty}^{i} \tilde{f}(w)(\cdot-w)^{k-2} \mathrm{~d} w+\int_{-i \infty}^{i} \stackrel{\circ}{f}(w)(\cdot-w)^{k-2} \mathrm{~d} w\right]_{2-k, 0}^{\|}(\gamma-1) .
\end{aligned}
$$

Since the term inside the square brackets belongs to $P_{k-2}(\mathbb{C})$, the cohomology class $j\left(\left[\phi^{\prime}\right](f)\right)$ of this cocycle coincides with $[\phi](f)$.

Remark In [2], (Th. 1.2.), it is proved that $\left[\phi^{\prime}\right]$ is an isomorphism.

We are now ready to prove

Theorem 6.3 Suppose that the class of $\in S_{k}^{!}$in $S_{k}^{!} / D^{k-1} M_{2-k}^{!}$is an eigenclass of the Hecke operators. Let $K_{f}$ denote the field generated by the Fourier coefficients of $f$. Then there are $\omega^{ \pm}(f) \in \mathbb{C}$ such that

$$
\begin{aligned}
& L_{f}^{*}(j) \in \omega^{+}(f) K_{f}, \text { for odd } j \in\{2, \ldots k-2\} \text { and } L_{f}^{*}(j) \in \omega^{-}(f) K_{f}, \\
& \quad \text { for even } j \in\{2, \ldots k-2\} .
\end{aligned}
$$

Proof In [12], it is proved that the eigenspace of the class of $f$ in $S_{k, \mathbb{Q}}^{!} / D^{k-1} M_{2-k, \mathbb{Q}}^{!} \otimes_{\mathbb{Q}}$ $\mathbb{C}$ is two-dimensional. It is defined over $K_{f}$. We let $V_{f}^{\text {deR }} \subset S_{k, \mathbb{Q}}^{!} / D^{k-1} M_{2-k, \mathbb{Q}}^{!} \otimes_{\mathbb{Q}} K_{f}$ 
denote the Hecke eigenspace generated over $K_{f}$ by $f$ and let $V_{f}^{B}$ be the corresponding eigenspace in $H^{1}\left(\Gamma, P_{k-2}(\mathbb{Q})\right) \otimes_{\mathbb{Q}} K_{f}$. (We follow the notation of [7] to indicate the de Rham- (resp. Betti)-cohomological origin of those eigenspaces.) The space $V_{f}^{B}$ is twodimensional and defined over $K_{f}$, and therefore, so is the corresponding eigenspace $V_{f}^{W}$ in $H_{\text {par }}^{1}\left(\Gamma, P_{k-2}(\mathbb{Q})\right) \otimes_{\mathbb{Q}} K_{f}$. It decomposes into invariant and anti-invariant eigenspaces with respect to the real Frobenius: $V_{f}^{W}=V_{f}^{W,+} \oplus V_{f}^{W,-}$. Further, by Theorem 6.1, the map

$$
\left[\phi^{\prime}\right]: V_{f}^{\text {deR }} \otimes_{K_{f}} \mathbb{C} \stackrel{\sim}{\rightarrow} V_{f}^{W} \otimes_{K_{f}} \mathbb{C}
$$

is a canonical isomorphism. Therefore, for some $\omega^{ \pm}(f) \in \mathbb{C}$, and some $\phi^{ \pm}(f) \in V_{f}^{W, \pm} \subset$ $H_{\text {par }}^{1, \pm}\left(\Gamma, P_{k-2}\left(K_{f}\right)\right)$, we have,

$$
\left[\phi^{\prime}(f)\right]=\omega^{+}(f) \phi^{+}(f)+\omega^{-}(f) \phi^{-}(f) .
$$

Thus, for an even $P^{+} \in Z_{\mathrm{par}}^{1}\left(\Gamma, P_{k-2}\left(K_{f}\right)\right)$, an odd $P^{-} \in Z_{\mathrm{par}}^{1}\left(\Gamma, P_{k-2}\left(K_{f}\right)\right)$ and a $c_{f} \in \mathbb{C}$,

$$
\phi^{\prime}(f)=\omega^{+}(f) P^{+}+\omega^{-}(f) P^{-}+c_{f} \psi_{0} .
$$

[Recall that $\psi_{0}$ is defined by (37)]. This gives

$$
\phi^{\prime}(f)(S)=\omega^{+}(f) P^{+}(S)+\omega^{-}(f) P^{-}(S)+c_{f} \underset{2-k, 0}{\|}(S-1) .
$$

On the other hand, it is easy to see from our definition of $L$-function and an application of the binomial formula to (39), that the coefficient of $z^{j-1}$ in $\phi^{\prime}(f)(S)$ is a multiple of $L_{f}^{*}(j)$ by an element of $\mathbb{Q}[i]$. By comparing, in (40), the coefficients of $z^{j-1}$, for $j$ odd (resp. even) other than $j=1$, and $j=k-1$, we deduce the assertion.

\section{Acknowledgements}

We thank F. Brown for many helpful comments on the exposition and possible further directions of research, K. Bringmann for suggesting an alternative approach to Theorem 1.5 and F. Strömberg for numerical tests of some of the results. This work was supported by Engineering and Physical Sciences Research Council (Grant No. EP/S032460/1).

\section{Conflict of interest}

On behalf of all authors, the corresponding author states that there is no conflict of interest.

Received: 4 April 2020 Accepted: 5 July 2020 Published online: 17 July 2020

\section{References}

1. Bringmann, K., Diamantis, N., Ehlen, S.: Regularized inner products and Eichler cocycles. Int. Math. Res. Not. 2017(24), 7420-7458 (2017)

2. Bringmann, K., Guerzhoy, P., Kent, Z., Ono, K.: Eichler-Shimura theory for mock modular forms. Math. Ann. 355 1085-1121 (2013)

3. Brown, F.: A class of non-holomorphic modular forms I. Res. Math. Sci. 5, 7 (2018)

4. Brown, F.: A class of non-holomorphic modular forms II: equivariant iterated Eisenstein integrals. Forum Math. Sigma 8, E31 (2020)

5. Brown, F.: A class of non-holomorphic modular forms III: real analytic cusp forms for $\mathrm{SL}_{2}$ (ZZ). Res. Math. Sci. 5, 34 (2018)

6. Brown, F.: Multiple modular values and the relative completion of the fundamental group of $M_{1,1}$. preprint. arXiv:1407.5167

7. Brown, F., Hain, R.: Algebraic de Rham theory for weakly holomorphic modular forms of level one. Algebra Number Theory 12(3), 723-750 (2018)

8. Bruinier, J., Funke, J.: On two geometric theta lifts. Duke Math. J. 125(1), 45-90 (2004)

9. Cohen, H., Strömberg, F.: Modular Forms. A Classical Approach. Graduate Studies in Mathematics, vol. 179. American Mathematical Society, Providence (2017)

10. Diamantis, N., Rolen, L.: Eichler cohomology and zeros of polynomials associated to derivatives of $L$-functions. J. Reine Angew. Math. (Crelle's J.) arXiv:1704.02667v1 (to appear)

11. Diamantis, N., O'Sullivan, C.: Kernels of L-functions of cusp forms. Math. Ann. 346(4), 897-929 (2010)

12. Guerzhoy, P.: Hecke operators for weakly holomorphic modular forms and supersingular congruences. Proc. Am. Math. Soc. 136, 3051-3059 (2008) 
13. Lewis, J.: Spaces of holomorphic functions equivalent to the even Maass cusp forms. Invent. Math. 127, 271-306 (1997)

14. Lewis, J., Zagier, D.: Period functions for Maass wave forms. I. Ann. Math. 153, 191-258 (2001)

15. Manin, Y.T.: Periods of parabolic points and p-adic Hecke series. Math. Sb. 21, 371-393 (1973)

16. Mühlenbruch, T.: Systems of automorphic forms and period functions. Thesis (2003)

\section{Publisher's Note}

Springer Nature remains neutral with regard to jurisdictional claims in published maps and institutional affiliations. 